\title{
chapter Biological Psychiatry in the UK and Beyond
}

\author{
Stephen Lawrie
}

\section{Introduction}

What is 'biological psychiatry'? With biology being the scientific study of life, if one took the word literally, one could legitimately question whether there is any other kind of psychiatry. ${ }^{1}$ By this definition, psychology is part of biology. As taught in schools and universities, however, biology is the more constrained study of living organisms and includes anatomy, physiology and behaviour; human biology includes all those aspects as well as genetics, anthropology and nutrition and so on. That is still quite broad.

What biological psychiatry is usually taken to mean is the search for neurobiological underpinnings of mental illness and application of drug and other physical treatments for them. This, of course, assumes that the brain-mind are sufficiently interlinked to justify that approach - something that is taken as read by most doctors and should be self-evident to anyone who has ever consumed any psychoactive drug, including caffeine and alcohol. What biological psychiatry does not (overtly) include are those key elements of human understanding that are the essential tools in the trade of the effective clinician: the application of insights from experience, perhaps informed by the arts and humanities, to the clinical encounter. There are, of course, those members of our broad church of psychiatry that prioritise psychosocial approaches to understanding and psychotherapy as treatment. To some of them, and many outside psychiatry, biological psychiatry is or at least can be reductionistic - reducing or ignoring the mind to little or nothing more than the brain. Anything so 'mindless' would be just as bad as dualism or mentalistic 'brainlessness'. ${ }^{2}$ One would, however, be hard-pressed to find any so-called or self-declared biological psychiatrist who does not pay heed to the importance of our mental lives.

\section{The Rise of Psychopharmacology}

In the 1940s, the therapeutic armamentarium available to psychiatrists included barbiturates and not much else. During the 1950s, cutting-edge neuroscience demonstrated the existence of neurotransmitters in the brain. Coincidentally, several new drugs were discovered, including tricyclic antidepressants, monoamine oxidase inhibitors (MAOIs), antipsychotics and lithium. One of the eminent pharmacologists of the age, John Henry Gaddum, was interested in LSD and proposed a role for serotonin (5-hydroxytryptamine, 5HT) in mood regulation. Gaddum was Professor of Pharmacology at the University of Edinburgh from 1942 to 1958 and in Cambridge from 1958 to 1965.

Two young psychiatrists working in Gaddum's departments, George Ashcroft and Donald Eccleston, proposed the monoamine theory of depression. The theory received initial support from a study that showed patients with depression had lower levels of the 
main 5HT metabolite 5-hydroxy indole acetic acid (5HIAA) in cerebrospinal fluid (CSF) than neurology patient 'controls' undergoing lumbar air encephalography. ${ }^{3}$ Strong support came from a study conducted in what was by then the Medical Research Council (MRC) Brain Metabolism Research Unit, in which CSF was sampled under standardised conditions - 5HIAA not only correlated with the severity of depression but normalised on remission. ${ }^{4}$ Less appealingly, levels were also low in those with schizophrenia (but not in mania). This apparent early success was further reinforced when Alec Coppen and colleagues at the MRC Neuropsychiatric Research Unit in Epsom, Surrey, showed that adding tryptophan (TRP, a 5HT precursor) to the antidepressant tranylcypromine helped get patients dramatically better, almost as effectively as electroconvulsive therapy (ECT). ${ }^{5}$

Decreased free and/or total TRP levels in the plasma and CSF in depressed patients were replicated in several labs, ${ }^{6}$ but Coppen was always concerned that it all could be a secondary change to depression and subsequent work by Ashcroft led him to the same conclusion. ${ }^{7}$ The weight loss and elevated cortisol of depression were just two of many possible confounders. ${ }^{8}$ On the other hand, several studies showed that rapid TRP and 5HT depletion - through, for example, ingesting a TRP-free amino acid drink - reduces mood in healthy volunteers and in those who are depressed or have recovered. This realisation led to extensive work on neuroendocrine disruptions in depression - particularly in Oxford - including demonstrations that hormonal responses were blunted in depression and normalised by some antidepressants and lithium, including ECT in patients and electroconvulsive stimulation in animals.

There is, of course, an analogous story to be told about the role of the adrenergic system in depression but the UK contribution to this was less central. Although there is no question that many treatments for depression act on the serotoninergic and other monoamine systems, it has not been established whether there is an abnormality of serotonin metabolism or that treatments correct it. It is more complicated than that. The 5HT system is probably modulating other processes critical to the development and maintenance of depression, such as adaptive responses to aversive events. ${ }^{9}$ Low 5HTIAA levels in CSF may mark severity and are associated with, and maybe even predictive of, impulsive, violent suicidal behaviour. This also seems to be true, however, of schizophrenia. ${ }^{10}$

Nevertheless, subsequent work employing functional neuroimaging as a window on the brain has shown that single and repeated doses of various antidepressants increase the recognition of happy facial expressions, and amygdala responses to them, while decreasing amygdala response to negative affect faces, in healthy people and in those with depression. ${ }^{11}$ These effects are also seen after seven days' administration in healthy participants and are maintained during longer-term treatment. Further, long-term administration of selective serotonin reuptake inhibitor (SSRI) or norepinephrine reuptake inhibitor antidepressants can enhance synaptic plasticity and block the synaptic and dendritic deficits caused by stress. $^{12}$

\section{Landmark Clinical Trials}

The advent of rigorous randomised controlled trials (RCTs) also coincided with the availability of many new drug treatments for depression, bipolar disorder and schizophrenia. Even if a simple monoamine theory of depression was not to survive, a series of landmark clinical trials carried out by psychopharmacologists and psychiatrists of various 
persuasions in the 1960s and 1970s established that antidepressants and other biological approaches to major psychiatric disorders worked.

The Clinical Psychiatry Committee of the MRC, which included epidemiologists like Archie Cochrane and Austin Bradford Hill, published its clinical trial of the treatment of depressive illness in the British Medical Journal in $1965 .{ }^{13}$ No fewer than 250 patients in London, Leeds and Newcastle aged 40-69 years with an untreated primary depressive illness (characterised by persistent low mood, with at least one of the following: morbid or delusional guilt, insomnia, hypochondriasis and psychomotor retardation or agitation) were randomised to ECT (4-8 treatments), $150 \mathrm{mg}$ imipramine, $45 \mathrm{mg}$ phenelzine or placebo over 4 weeks. About one-third of those on placebo improved notably but this was almost doubled in those on imipramine and more than doubled in those given ECT - and these differences were maintained at six months. Moreover, in those who had responded to imipramine, continuation with 75-150 mg over a further six-month period meant that only 22 per cent relapsed as compared to 50 per cent randomised to placebo. ${ }^{14}$

During the 1960s, Baastrup and Schou working independently and then together in Denmark, conducting studies that suggested lithium was effective in acute mania and had prophylactic properties. However, to Aubrey Lewis and Michael Shepherd in the MRC Social Psychiatry Unit at the Institute of Psychiatry (IOP) in London, lithium was 'dangerous nonsense' and 'a therapeutic myth', which, in their opinion, was based on 'serious methodological shortcomings' and 'spurious claims' (see also Chapters 2 and 17). ${ }^{15}$ Schou and Baastrup undertook a double-blind discontinuation trial with patients with 'manicdepressive illness' successfully treated with lithium who were then randomly allocated to continue on lithium or placebo. Lithium was superior in preventing relapse - but only in typical cases. ${ }^{16}$ Coppen and colleagues randomised sixty-five patients with recurrent affective disorders to lithium or identical-looking placebo in four centres for up to two years 86 per cent of those on lithium (0.73-1.23 meq per litre) were judged by independent psychiatrists and psychiatric social workers to have had no further episodes over that time, as compared to 8 per cent of the placebo group. ${ }^{17}$ What is more, lithium seemed to be equally effective in unipolar and bipolar patients.

Following observations that the turnover of 5HT was greatly increased by the administration of the amino acid TRP, Donald Eccleston (having moved to Newcastle) led the introduction of a new '5HT cocktail' or 'Newcastle cocktail' therapy for severe depression. Using l-tryptophan alone or combined with other drugs, such as phenelzine (or clomipramine) and lithium, frequently produced dramatic improvement in otherwise chronically treatment-resistant depressed patients. ${ }^{18}$

The landmark study of the use of antipsychotic drugs in acute schizophrenia was carried out with more than 400 patients admitted to 9 centres around the United States, about a half of whom were in their first episodes. ${ }^{19}$ By the end of the trial, 75 per cent of the patients receiving antipsychotic showed moderate or marked improvement, whereas only about 23 per cent did on placebo. It was left, however, to British social and biological psychiatrists to robustly demonstrate that these drugs also reduced the risk of relapse in the longer term whether with oral medication or depot long-acting intramuscular injection. ${ }^{20}$

\section{Innovative British Neuroimaging}

The independent realisation that X-ray intensity reduction by the brain could be accurately measured and reconstructed into a brain image earned Allan Cormack (Tufts University) 
and Godfrey Hounsfield (Electric \& Musical Industries (EMI), Middlesex) the Nobel Prize for Physiology/Medicine in 1979 for the development of computer assisted tomography. This was all the more remarkable as Hounsfield had gone to work for EMI immediately after school, making him the first person to win a Nobel Prize without going to university since Albert Einstein. Computerised tomography (CT), as it came to be known, became available for clinical use in 1971. Demonstration systems for CT of the head were installed in Glasgow, London and Manchester and the first body scanner (the CT5000) was installed for research at the Northwick Park Hospital (NPH) MRC Clinical Research Centre (CRC) on the outskirts of London in 1975.

Tim Crow was Head of the CRC Division of Psychiatry and intrigued that many patients with schizophrenia had cognitive impairment. He gave the young Glaswegian émigré Eve Johnstone the task of using CT to see if this might have an organic basis. Mid-axial brain slice photographs were traced three times each to calculate an average lateral ventricle-tobrain ratio (VBR), which was markedly increased in the patients. ${ }^{21}$ One can imagine how the finding that schizophrenia - a 'functional' psychosis - might have an organic basis was greeted by social psychiatrists, psychotherapists and neurologists at the time. Indeed, the copy of the paper in the Lancet in the IOP library reputedly had 'Rubbish' scrawled across it. Regardless, the finding was widely replicated, as was the association with cognitive impairment. $^{22}$

Important work contributing to the development of magnetic resonance imaging (MRI) as a non-invasive means of imaging brain and body in greater detail was done in Aberdeen by John Mallard and in Nottingham by Peter Mansfield (for which he was to share the 2003 Nobel Prize with Paul Lauterbur of Illinois, United States). US business and researchers capitalised upon this and the landmark MRI studies in schizophrenia were done there. ${ }^{23}$ It was clear by the turn of the millennium that people with schizophrenia had reduced whole brain volumes and additional decrements in parts of the prefrontal and temporal lobes. ${ }^{24}$ Further, there is a consistent association between these reductions and negative and positive symptoms respectively.

This work stimulated a resurgence of interest in the neuropathology of mental illness, especially schizophrenia, which provides independent confirmation of the findings and suggests they derive from the reduced density of neurons and glia, and lesser dendritic arborisation. ${ }^{25}$ These could, of course, partly derive from antipsychotic medication, as well as the other effects of long-term illness and alcohol excess. However, the demonstration of similar but lesser changes in relatives and first episode cases, ${ }^{26}$ and in those at elevated clinical risk ${ }^{27}$ with further reductions as some develop schizophrenia, has opened the way to potentially using neuroimaging to predict schizophrenia - which remains a very active global research effort.

\section{Functional Neuroimaging}

The first robust evidence that patients with schizophrenia had 'hypofrontality' - underactive prefrontal lobes - came from Ingvar and Franzén at the Karolinksa Institute in Sweden. ${ }^{28}$ The British contribution was to rather undermine confidence in this finding. Researchers in Edinburgh and London demonstrated that hypofrontality was more anatomically constrained, that it could also be found in depression and even that 'hyperfrontality' could be found in unmedicated first episode patients. ${ }^{29}$ Consequently, a Lancet editorial could pronounce 'Hypofrontality RIP' in 1995. 
Following Chris Frith's lead, several important positron emission tomography (PET) studies at the MRC Cyclotron Unit at the Hammersmith Hospital in London showed a complex but compelling picture of neurofunctional correlates of symptoms, especially of auditory-verbal hallucinations. These findings suggested that such hallucinations are associated with under-activation of language areas in the brain concerned with the monitoring of inner speech. ${ }^{30}$ These insights depended upon a technique that could analyse whole-brain tracer data. Karl Friston not only invented a Statistical Parametric Mapping procedure to do this but also would apparently stay up all night adding functions if one was needed for a particular analysis.

The Functional Imaging Laboratory (FIL) was founded in 1994, within the Institute of Neurology, following a major grant award from the Wellcome Trust. It pioneered new neuroimaging techniques such as functional MRI (fMRI) for understanding human cognition. Generously and wisely, Friston made his 'SPM' programme for analysing these data freely available and supported from the FIL and it remains the industry standard worldwide. The combination of fMRI and SPM facilitated more sophisticated studies to map auditory hallucinations, to relate them to dysconnectivity between language regions in the brain and to integrate these findings with dopamine signalling.

\section{Dopamine}

Two independent North American groups demonstrated in the early 1970s that the clinical potencies of antipsychotic drugs very strongly correlated with their ability to inhibit tritiated $(3 \mathrm{H})$ dopamine binding to postsynaptic receptors in mammalian brain samples. Clinical trials at NPH reinforced a dopaminergic theory of schizophrenia but also showed that it applied to other psychotic disorders. ${ }^{31}$ Some post-mortem studies also showed that the binding of $3 \mathrm{H}$-labelled spiroperidol was increased in parts of the basal ganglia and amygdala, but other studies suggested it was secondary to drug treatment. An early PET study with spiroperidol $77 \mathrm{Br}$-brominated to emit gamma rays found an increase in activity in drug-free patients, but this too was disputed - with sometimes heated exchanges between the labs leading this work at Johns Hopkins and the Karolinksa.

It was only with the development of another tracer - fluorodopa - which is incorporated into dopamine and therefore measures dopamine synthesis and turnover that the dopamine story in schizophrenia has been clarified. Using fluorodopa PET, researchers at Imperial and Kings Colleges in London have shown that young people at high clinical risk of psychosis have elevated dopamine turnover in the striatum, which correlates with psychotic (but not other) symptoms, is highest in those most likely to become ill and increases as they develop a psychotic disorder. ${ }^{32}$ (It should be noted, however, that there is a similarly strong strand of evidence that glutamatergic neurotransmission is also disrupted in schizophrenia.)

\section{A Note on Dementia Imaging}

Psychiatrists of many persuasions were among the vanguard of researchers using early neuroimaging techniques to study morphological and perfusion pattern changes in the brain in Alzheimer's disease and to distinguish them from those in multi-infarct dementia and from normal controls. Indeed, a generation of Old Age psychiatrists - inspired by Martin Roth in Newcastle and then Cambridge - did much to develop wider scientific and clinical interest in these conditions. As neurologists became more interested, they established that brain atrophy can be visualised by CT or MRI and that serial imaging and 
quantifying the degree of atrophy could aid diagnosis. Indeed, CT or MRI is now routinely recommended in many clinical guidelines in the evaluation of possible dementia and is now included in some diagnostic criteria. Further, Ian McKeith and John O'Brien have led the application of the accurate and reliable measure of low dopamine transporter activity in the brain in making a diagnosis of Lewy body dementia as distinct from others. ${ }^{33}$

\section{The Cochrane Collaboration and Evidence-Based Medicine}

The Cochrane Collaboration was founded in 1993 in response to Archie Cochrane's earlier call for up-to-date, systematic reviews of all relevant RCTs across health care (see also Chapter 17). Many academic and clinical psychiatrists from different specialties were early and enthusiastic contributors, and dedicated groups for schizophrenia and dementia were among the first to get established and publish reviews.

It quickly became evident that the RCT literature in psychiatry was about as good or bad as it was in most of medicine - with the notable exceptions of neurology and cardiology - in that there were far too many small, short and poorly reported trials. Nonetheless, systematic reviews and meta-analyses of the best available evidence showed that antidepressants and antipsychotics successfully treated acute depression or schizophrenia and that continuing effective treatment for a year compared with treatment discontinuation reduced relapse rates from around 41 per cent to 18 per cent for depression (31 RCTs, 4,410 participants) ${ }^{34}$ and from 64 per cent to 27 per cent for schizophrenia (65 RCTs, 6,493 patients). ${ }^{35}$ These differences of 23 per cent and 37 per cent mean that, on average, about one in three or four patients will benefit from taking these drugs over a year - and these are some of the largest treatment effects in the whole of medicine. There was even RCT evidence (32 RCTs, 3,458 patients) that lithium reduced suicide and overall mortality, although these were based on (fortunately) small numbers of deaths. ${ }^{36}$ The UK ECT review group included service users and established that real ECT was significantly and substantially more effective than simulated or sham ECT (6 RCTs, 256 patients, all done in the UK in the 1970s) and more effective than pharmacotherapy (18 trials, 1,144 participants). ${ }^{37}$

Cochrane, as it has become known, and the wider rise of what might be called the evidence-based medicine movement came at roughly the same time as the development and aggressive marketing of the new 'atypical' antipsychotics (and valproate and various antidepressants) as more effective and/or better tolerated than the old drugs. Varying definitions of atypicality, study populations, outcomes and the reporting of these meant that wellconducted RCTs could show that 'olanzapine beats risperidone, risperidone beats quetiapine, and quetiapine beats olanzapine' and that all were, of course, better than the standard comparator drug haloperidol (as was required for FDA approval). Even if some or all of the apparent benefits were down to these new antipsychotics being used at lower doses than psychiatrists had got into the bad habit of using when prescribing older drugs ${ }^{38}$ Big Pharma had realised this. There was a clear need for independently funded and conducted RCTs.

This realisation led to the Clinical Antipsychotic Trials in Intervention Effectiveness (CATIE) study, which is probably the largest and most expensive clinical trial ever done in schizophrenia. It cost the US taxpayer the best part of $\$ 100$ million. Lieberman and colleagues randomised 1,493 patients at 57 US sites to one of five treatments. ${ }^{39}$ The primary outcome measure of continuing medication was only achieved in 26 per cent of people at eighteen months but this was about 10 per cent higher in patients allocated to take olanzapine - even if they also tended to put on weight and suffer metabolic derangements. 
In the UK, the CUtLASS trial showed similarly slight, if any, advantages of the newer antipsychotics, ${ }^{40}$ while the BALANCE trial showed that lithium was superior to valproate in preventing relapse in bipolar disorder. ${ }^{41}$ Systematic reviews showed that the new antidepressant, mood stabilising and antipsychotic drugs did not have simple class effects and each drug had subtle differences in terms of reducing certain symptoms and causing various adverse effects.

\section{Laying the Groundwork and Going Global for Genetic Advances}

It has long been known that major psychiatric disorders aggregate in families. This was conclusively demonstrated for schizophrenia by Gottesman and Shields in 1966, ${ }^{42}$ while working at MRC Psychiatric Genetics Unit at the IOP, with the assistance of Elliot Slater. He had kept records of twins of whom at least one had a diagnosis of schizophrenia. Taken together with data from eleven earlier major twin studies, an identical twin was at least forty times more likely to have schizophrenia than a person from the general population and a fraternal twin of the same sex around nine to ten times as likely. These data strongly suggested a strong genetic basis for schizophrenia and adoption studies outside the UK proved it.

Ongoing twin studies at the IOP established beyond doubt that the heritability of schizophrenia, schizoaffective disorder and mania were substantial and similar (82-85 per cent). What was controversial was the mode of inheritance - whether it was due to a small number of rare but highly penetrant mutations or more attributable to polygenic liability in a diathesis stress-model. The identification of a chromosomal translocation from $t 1: 11$ in a large Scottish family in 1990 led to the identification of the 'DISC1' (Disrupted in Schizophrenia 1) gene. ${ }^{43}$ The association was, however, strongest when the mental disorders in the phenotype included recurrent major depression and adolescent conduct and emotional disorders. Even though this family may be unique and common variants in DISC1 are not (at least as yet) identified as risk factors for any specific disorder, this discovery kept the field going during the long lean years of non-replicated linkage and association studies.

What was to transform psychiatric genetics was the Human Genetics Project (HGP). This started in 1990, funded by the US Department of Energy and the US National Institutes of Health and supported by the Wellcome Trust through the Sanger Centre in Cambridge. The first draft of the complete sequence of nucleotides in the human genome was published in 2001 and launched modern human genetics. The identification of rare, penetrant genetic variants causing monogenic diseases boomed in the following years and paved the way for the systematic screening of disease genes in diagnostic services - including those with severe learning disability. The HGP also brought about advances in technology, particularly 'next-generation sequencing', which led to the first available arrays for genome-wide association studies (GWAS).

The early psychiatric GWAS did not lead to significant findings, which led to some losing faith in the approach. Others persisted, and in one of the first and best examples of collaboration science the Wellcome Trust Case Control Consortium published (in 2007) the then largest GWAS to date and set the scene for the spate of gene discovery that was to follow. They examined approximately 2,000 individuals for each of seven major diseases and a shared set of approximately 3,000 controls and identified 24 independent association signals including one in bipolar disorder (and 1-9 in coronary artery disease, rheumatoid arthritis, type 1 and type 2 diabetes and Crohn's disease). ${ }^{44}$

The Psychiatric Genomics Consortium was also formed in 2007, which allowed thousands of samples from all over the world to be shared. This collaboration quickly delivered 
the first significant findings from GWAS for schizophrenia, as well as evidence that major psychiatric disorder was very highly polygenic. ${ }^{45}$ Nevertheless, some rare mutations of large effect were clearly implicated in neurodevelopmental disorders such as autism, attention deficit hyperactivity disorder (ADHD) and schizophrenia. ${ }^{46}$

It has become increasingly clear in the past decade that GWAS is a numbers game. Pooling 100,000 cases of schizophrenia and controls led to no fewer than 108 schizophreniaassociated genetic loci becoming evident. ${ }^{47}$ Adding another 35,000 people identified another 37 'hits' and more are on the way. Indeed, the success of GWAS in schizophrenia has led to it being called the poster child of the GWAS generation. Bipolar disorder and depression are now yielding their genetic secrets too.

It is, however, equally clear that the genes identified are pleiotropic - that is, they have multiple effects and so do not map neatly on to specific disorders. Just as the rare mutations increase the risk for a variety of conditions, the risk variants for common psychiatric disorders overlap to a large extent. Nonetheless, there are likely to be some specific genes and biological pathways as well as others cutting across disorders. Although such insights have yet to lead to innovations in the clinical management of patients, they certainly have promise for diagnostics and therapeutics.

\section{The Decade of the Brain and the Next Ten Years}

While the psychiatric geneticists have been trailblazing, the neuroimaging research community have organised themselves into large global consortia employing common and increasingly innovative methods. Most notably, the Enhancing Imaging Genetics through Meta-Analysis (ENIGMA) consortium have combined data from thousands of scans which have confirmed and strengthened the results from previous studies and meta-analyses and delivered novel insights into the genetics of neuroimaging measures. The application of mathematical graph theory tools to neuroimaging data provides a way of studying neural systems efficiency at a whole-brain (connectome) level.

Most excitingly, contemporary neuroscience and philosophy see the brain-mind (after Reverend Thomas Bayes) as testing hypotheses about the world, from previous experience, against ongoing experience and updating the inner model of the world as required. ${ }^{48}$ In essence, structural and functional disturbances of fronto-temporal brain systems could reduce their reliable co-ordinated input, disrupt reality testing and impair the use of memories to guide perception and action. Most of the research thus far has been done on schizophrenia with some replicated findings if not yet a true consensus - but this and other forms of 'computational psychiatry' offer objective measures of otherwise subjective impressions that promise to be revealing across psychiatry and indeed neuroscience as a whole. ${ }^{49}$

It has also become clear in the last ten years that the structural and functional neuroimaging findings in various disorders overlap to a large degree. To some extent, this is hardly surprising given the overlap in genetic and environmental risk factors and the comorbidities of mental disorders. The increasing incorporation of psychosocial risk markers - such as the role of personality, childhood adversity and stress and their biological correlates - into multivariate risk models of mental illness alongside polygenic risk scores and machine learning approaches to data analysis will advance progress towards clinical applications. Despite these complexities, there has been notable progress in developing neuroimaging biomarkers of depression and schizophrenia in the 2010s. ${ }^{50}$ 


\section{Conclusion}

This has, of necessity, been a relatively brief and focused review of fifty years and more of research endeavour. It has also been positive in stressing replicated advances and ignoring less profitable research streams, such as the red herring of the 'pink spot' in schizophrenia. Equally, however, British psychiatrists have made major contributions to understanding and treating many conditions - including autism, ADHD, anxiety and alcohol and drug dependence - which we have not had the space to do justice to.

Overall, it is difficult to avoid the conclusion that 'biological psychiatry' has been a success. Indeed, the historian Edward Shorter said as much as far back as $1999 .^{51}$ However, it makes little sense to talk of a biological psychiatry pursued by biological psychiatrists. It is simply medicine done by doctors who specialise in the diagnosis and management of mental illness.

Disquietingly, far too many psychiatrists seem unaware that drug treatments in psychiatry are about as good as in the rest of medicine. ${ }^{52}$ As for the research, we should redouble our efforts to find biomarkers of diagnosis and in particular of treatment response. This is within our grasp if the field receives the research funding that reflects the societal costs of the conditions we deal with. This is also what the patients with these conditions and their carers want, as the James Lind Alliance (JLA) has demonstrated. The JLA, whose infrastructure is funded by the UK National Institute for Health Research (NIHR), brings patients, carers and clinicians together, in Priority Setting Partnerships, to identify and prioritise unanswered questions. There is a remarkable convergence of interests in, for example, determining causes, better diagnosis, early interventions, personalised approaches and better treatments with fewer adverse effects.

\section{Key Summary Points}

- Many scientists, academic and clinical psychiatrists have contributed to the search for the biological basis of mental illness, leading to many notable discoveries and particular advances in understanding schizophrenia.

- RCTs have established beyond reasonable doubt the efficacy of antidepressants, ECT, antipsychotics and mood stabilisers.

- The most striking diagnostic advances have been made in identifying the genetics of learning disability and in developing neuroimaging and blood-based biomarkers of dementia.

- Polygenic risk scores and machine learning of neuroimaging and other data have real potential to impact upon clinical practice and improve patient care.

- Psychiatrists should join those affected by mental illness in calling for increased funding to identify biomarkers, develop new treatments and improve services.

\section{Notes}

1. S. B. Guze, Biological psychiatry: Is there any other kind? Psychological Medicine (1989) 19: 315-23.

2. L. Eisenberg, Mindlessness and brainlessness in psychiatry. British Journal of Psychiatry (1986) 148: 497-508.

3. G. W. Ashcroft and D. F. Sharman, 5-Hydroxyindoles in human cerebrospinal fluids. Nature (1960) 186: $1050-1$. 
4. G. W. Ashcroft, T. B. Crawford, D. Eccleston et al., 5-hydroxyindole compounds in the cerebrospinal fluid of patients with psychiatric or neurological diseases. The Lancet (1966) 2: 1049-52.

5. A. Coppen, D. M. Shaw and J. P. Farrell, Potentiation of the antidepressive effect of a monoamine-oxidase inhibitor by tryptophan. The Lancet (1963) 1: 79-81.

6. A. Coppen, E. G. Eccleston and M. Peet, Total and free tryptophan concentration in the plasma of depressive patients. The Lancet (1973) 2: 60-3.

7. E. Shorter, How Everyone Became Depressed. Oxford: Oxford University Press: 2013.

8. B. J. Carroll, M. Feinberg, J. F. Greden et al., A specific laboratory test for the diagnosis of melancholia: Standardization, validation, and clinical utility. Archives of General Psychiatry (1981) 38: 15-22.

9. J. F. W. Deakin and F. G. Graeff, 5-HT and mechanisms of defence. Journal of Psychopharmacology (1991) 5: $305-15$.

10. S. J. Cooper, C. B. Kelly and D. J. King, 5-Hydroxyindoleacetic acid in cerebrospinal fluid and prediction of suicidal behaviour in schizophrenia. The Lancet (1992) 340: 940-1.

11. C. J. Harmer, R. S. Duman and P. J. Cowen, How do antidepressants work? New perspectives for refining future treatment approaches. Lancet Psychiatry (2017) 4: 409-18.

12. Ibid.

13. British Medical Research Council, Clinical trial of the treatment of depressive illness. British Medical Journal (1965) 1: 881-6.

14. R. H. Mindham, C. Howland and M. Shepherd, Continuation therapy with tricyclic antidepressants in depressive illness. The Lancet (1972) 2: 854-5.

15. E. Shorter, The history of lithium therapy. Bipolar Disorders (2009) 11(Suppl 2): 4-9.

16. P. C. Baastrup, J. C. Poulsen, M. Schou, K. Thomsen and A. Amdisen, Prophylactic lithium: Double blind discontinuation in manic-depressive and recurrent-depressive disorders. The Lancet (1970) 2: 326-30.

17. A. Coppen, R. Noguera and J. Bailey, Prophylactic lithium in affective disorders: Controlled trial. The Lancet (1971) 2: 275-9.

18. W. A. Barker, J. Scott and D. Eccleston, The Newcastle chronic depression study: Results of a treatment regime. International Clinical Psychopharmacology (1987) 2: 261-72.

19. National Institute of Mental Health Psychopharmacology Service Center Collaborative Study Group, Phenothiazine treatment of acute schizophrenia: Effectiveness. Archives of General Psychiatry (1964) 10: $246-61$.

20. J. P. Leff and J. K. Wing, Trial of maintenance therapy in schizophrenia. British Medical Journal (1971) 3: 599-604; S. R. Hirsch, R. Gaind, P. D. Rohde, B. C. Stevens and J. K. Wing, Outpatient maintenance of chronic schizophrenic patients with long-acting fluphenazine: Double-blind placebo trial. Report to the Medical Research Council Committee on Clinical Trials in Psychiatry. British Medical Journal (1973) 1: 633-7.

21. E. C. Johnstone, T. J. Crow, C. D. Frith, J. Husband and L. Kreel, Cerebral ventricular size and cognitive impairment in chronic schizophrenia. The Lancet (1976) 2: 924-6.

22. S. W. Lewis, Computerised tomography in schizophrenia 15 years on. British Journal of Psychiatry (1990) 157 (Suppl 9): 16-24.

23. R. L. Suddath, G. W. Christison, E. F. Torrey, M. F. Casanova and D. R. Weinberger. Anatomical abnormalities in the brains of monozygotic twins discordant for schizophrenia. New England Journal of Medicine (1990) 322: 789-94.

24. S. M. Lawrie and S. S. Abukmeil, Brain abnormality in schizophrenia: A systematic and quantitative review of volumetric magnetic resonance imaging studies. British Journal of Psychiatry (1998) 172: 110-20.

25. P. J. Harrison, N. Freemantle and J. R. Geddes, Meta-analysis of brain weight in schizophrenia. Schizophrenia Research (2003) 64: 25-34. 
26. S. M. Lawrie, H. Whalley, J. N. Kestelman et al., Magnetic resonance imaging of brain in people at high risk of developing schizophrenia. The Lancet (1999) 353: 30-3.

27. C. Pantelis, D. Velakoulis, P. D. McGorry et al., Neuroanatomical abnormalities before and after onset of psychosis: A cross-sectional and longitudinal MRI comparison. The Lancet (2003) 361: 281-8.

28. D. H. Ingvar and G. Franzén, Distribution of cerebral activity in chronic schizophrenia. The Lancet (1974) 2: 1484-6.

29. K. P. Ebmeier, S. M. Lawrie, D. H. Blackwood, E. C. Johnstone and G. M. Goodwin, Hypofrontality revisited: A high resolution single photon emission computed tomography study in schizophrenia. Journal of Neurology, Neurosurgery, and Psychiatry (1995) 58: 452-6.

30. P. K. McGuire, D. A. Silbersweig, I. Wright et al., Abnormal monitoring of inner speech: A physiological basis for auditory hallucinations. The Lancet (1995) 346: 596-600.

31. E. C. Johnstone, T. J. Crow, C. D. Frith, M. W. Carney and J. S. Price, Mechanism of the antipsychotic effect in the treatment of acute schizophrenia. The Lancet (1978) 1: 848-51.

32. O. D. Howes, S. K. Bose, F. Turkheimer et al., Dopamine synthesis capacity before onset of psychosis: A prospective [18 F]-DOPA PET imaging study. American Journal of Psychiatry (2011) 168: 1311-17.

33. I. McKeith, J. O’Brien, Z. Walker et al., Sensitivity and specificity of dopamine transporter imaging with 123I-FPCIT SPECT in dementia with Lewy bodies: A phase III, multicentre study. Lancet Neurology (2007) 6: 305-13.

34. J. R. Geddes, S. M. Carney, C. Davies et al., Relapse prevention with antidepressant drug treatment in depressive disorders: a systematic review. The Lancet (2003) 361: 653-61.

35. S. Leucht, M. Tardy, K. Komossa et al., Antipsychotic drugs versus placebo for relapse prevention in schizophrenia: A systematic review and meta-analysis. The Lancet (2012) 379: 2063-71.

36. A. Cipriani, H. Pretty, K. Hawton and J. R. Geddes, Lithium in the prevention of suicidal behavior and all-cause mortality in patients with mood disorders: A systematic review of randomized trials. American Journal of Psychiatry (2005) 162: 1805-19.

37. UK ECT Review Group, Efficacy and safety of electroconvulsive therapy in depressive disorders: A systematic review and meta-analysis. The Lancet (2003) 361: 799-808.

38. J. Geddes, N. Freemantle, P. Harrison and P. Bebbington, Atypical antipsychotics in the treatment of schizophrenia: Systematic overview and meta-regression analysis. British Medical Journal (2000) 321(7273): 1371-6.

39. J. A. Lieberman, T. S. Stroup, J. P. McEvoy et al., Effectiveness of antipsychotic drugs in patients with chronic schizophrenia. New England Journal of Medicine (2005) 353: 1209-23.

40. P. B. Jones, T. R. Barnes, L. Davies et al., Randomized controlled trial of the effect on Quality of Life of secondvs first-generation antipsychotic drugs in schizophrenia: Cost Utility of the Latest Antipsychotic Drugs in Schizophrenia Study (CUtLASS 1). Archives of General Psychiatry (2006) 63: 1079-87.

41. BALANCE investigators and collaborators, J. R. Geddes, G. M. Goodwin et al., Lithium plus valproate combination therapy versus monotherapy for relapse prevention in bipolar I disorder (BALANCE): A randomised open-label trial. The Lancet (2010) 375: 385-95.

42. I. I. Gottesman and J. Shields, Schizophrenia in twins: 16 years' consecutive admissions to a psychiatric clinic. British Journal of Psychiatry (1996) 112: 809-18.

43. D. St Clair, D. Blackwood, W. Muir et al., Association within a family of a balanced autosomal translocation with major mental illness. The Lancet (1990) 336: 13-16.

44. Wellcome Trust Case Control Consortium, Genome-wide association study of 14,000 cases of seven common diseases and 3,000 shared controls. Nature (2007) 447: 661-78.

45. International Schizophrenia Consortium, S. M. Purcell, N. R. Wray et al., Common polygenic variation contributes to risk of schizophrenia and bipolar disorder. Nature (2009) 460: 748-52.

46. N. M. Williams, I. Zaharieva, A. Martin et al., Rare chromosomal deletions and duplications in attention-deficit hyperactivity disorder: A genome-wide analysis. The Lancet (2010) 376: 1401-8. 
47. Schizophrenia Working Group of the Psychiatric Genomics Consortium, Biological insights from 108 schizophrenia-associated genetic loci. Nature (2014) 511: 421-7.

48. P. C. Fletcher and C. D. Frith, Perceiving is believing: A Bayesian approach to explaining the positive symptoms of schizophrenia. Nature Reviews Neuroscience (2009) 10: 48-58.

49. K. J. Friston, K. E. Stephan, R. Montague and R. J. Dolan, Computational psychiatry: The brain as a phantastic organ. Lancet Psychiatry (2014) 1: 148-58.

50. A. T. Drysdale, L. Grosenick, J. Downar et al., Resting-state connectivity biomarkers define neurophysiological subtypes of depression. Nature Medicine (2017) 23: 28-38; A. Li, A. Zalesky, W. Yue et al., A neuroimaging biomarker for striatal dysfunction in schizophrenia. Nature Medicine (2020) 26: 558-65.

51. E. Shorter, A History of Psychiatry: From the Era of the Asylum to the Age of Prozac. New York: Wiley and sons, 1997, p. vii.

52. S. Leucht, S. Hierl, W. Kissling, M. Dold and J. M. Davis, Putting the efficacy of psychiatric and general medicine medication into perspective: Review of meta-analyses. British Journal of Psychiatry (2012) 200: 97-106. 